\title{
Resonant tunneling via spin-polarized barrier states in a magnetic tunnel junction
}

\author{
R. Jansen and J. C. Lodder \\ $M E S A+$ Research Institute, University of Twente, 7500 AE Enschede, The Netherlands
}

(Received 1 September 1999)

\begin{abstract}
Resonant tunneling through states in the barrier of a magnetic tunnel junction has been analyzed theoretically for the case of a spin-polarized density of barrier states. It is shown that for highly spin-polarized barrier states, the magnetoresistance due to resonant tunneling is enhanced compared to the magnetoresistance due to direct tunneling between the ferromagnetic electrodes. Moreover, it is predicted that whenever the barrier states have a nonzero polarization, tunneling magnetoresistance exists even if only one electrode is magnetic, while the other is not. The above effects have been studied for arbitrary location of the states. In particular, we consider the case of a thin sheet of magnetic dopants in close proximity to and coupled ferromagnetically or antiferromagnetically to one of the electrodes. Depending on the dopant polarization, the distance to the electrode and the type of coupling, the total magnetoresistance can be enhanced or reduced, and may be reversed for antiferromagnetic coupling.
\end{abstract}

\section{INTRODUCTION}

The process of resonant tunneling, in which the transmission coefficient of a double barrier system has a sharply peaked maximum at the energy of a quasibound state, has been observed in several systems since pioneering experiments by Tsu and Esaki. ${ }^{1,2}$ This phenomenon, that gives rise to negative differential resistance, is discussed in the context of semiconductor heterostructures in many textbooks on lowdimensional semiconductors. ${ }^{3,4}$ Resonant tunneling has also been observed in metallic tunneling structures, ${ }^{5,6}$ predominantly in junctions having only a single tunnel barrier formed by an amorphous material like $\mathrm{Si}$ or $\mathrm{Ge}^{5,7}$ In that case, defect levels intrinsic to the barrier material provide the resonant levels, thereby effectively splitting the tunnel barrier into two parts. Experimental ${ }^{8}$ and theoretical ${ }^{9,10}$ studies have included the evolution from resonant tunneling through a single barrier state, to multistep tunneling and even variable range hopping as the barrier thickness is increased. ${ }^{8}$ Also, inelastic processes like phonon absorption/emission have been considered. ${ }^{6,10}$

The use of ferromagnetic electrodes in tunneling structures ${ }^{1-13}$ has added a new dimension to resonant tunneling, namely the electron spin. The effect of impurityassisted tunneling on the magnetoresistance of a magnetic tunnel junction was theoretically studied by Bratkovsky, ${ }^{14}$ in a single-resonance approximation and a uniform distribution of resonant energies. Bratkovsky showed that, because tunneling through impurity levels in the barrier is governed by the overlap of the electrode wave functions with the unpolarized barrier states, the effective spin polarization for this process is reduced as compared to that of electrons tunneling directly between the magnetic electrodes. A similar conclusion was obtained by Tsymbal and Pettifor. ${ }^{15}$

The calculated reduction of tunneling polarization has been confirmed experimentally via the controlled introduction of dopants like Si ions into the barrier of magnetic tunnel junctions. ${ }^{16,17}$ However, for the specific case of Fe ions in an $\mathrm{Al}_{2} \mathrm{O}_{3}$ barrier, the magnetoresistance was found to be enhanced. ${ }^{18}$ This has led us to consider the possibility of resonant tunneling through barrier levels that have a spindependent density. In this paper, we theoretically evaluate resonant tunneling through such spin-polarized barrier states. First, we shall generalize the model of Bratkovsky to include the spin polarization of the levels, and show that the associated resonant-tunneling magnetoresistance can indeed be enhanced depending on the polarization of the barrier states. Second, we show that whenever the states have a nonzero spin polarization, tunneling magnetoresistance exists even if only one electrode is magnetic, while the other is not. Third, we apply the formalism to the case of a thin sheet of magnetic dopants in close proximity to and coupled ferromagnetically or antiferromagnetically to one of the magnetic electrodes. We find that the total tunnel magnetoresistance can be enhanced, reduced and, for antiferromagnetic coupling, can be reversed.

\section{MODEL DESCRIPTION}

The conductance due to resonant or impurity-assisted tunneling of electrons with spin $\sigma$ is given by ${ }^{14}$

$$
G_{\sigma}=\frac{2 e^{2}}{\pi \hbar} \sum_{i} \frac{\Gamma_{l \sigma} \Gamma_{r \sigma}}{\left(E_{i \sigma}-\mu\right)^{2}+\Gamma_{\sigma}^{2}},
$$

where $\Gamma_{l \sigma}$ and $\Gamma_{r \sigma}$ are the partial widths of the resonance corresponding to tunneling of an electron with spin $\sigma$ from the barrier state to the left or right magnetic electrode, respectively. $\Gamma_{\sigma}=\Gamma_{l \sigma}+\Gamma_{r \sigma}$ is the total width of the resonance, while $\left(E_{i \sigma}-\mu\right)$ is the energy of the barrier level with respect to the Fermi level $\mu$. We will consider a rectangular barrier of height $\phi$ and total width $w$ between two not necessarily identical magnetic electrodes characterized by spindependent Fermi wave vectors $k_{l \sigma}$ and $k_{r \sigma}$. For a state located at a distance $w_{l}\left(w_{r}=w-w_{l}\right)$ from the left (right) electrode, the partial widths are given by

$$
\Gamma_{l \sigma}=\phi \frac{2 m k_{l \sigma}}{\kappa_{0}^{2}+m^{2} k_{l \sigma}^{2}} \frac{e^{-2 \kappa_{0} w_{l}}}{\kappa_{0} w_{l}},
$$




$$
\Gamma_{r \sigma}=\phi \frac{2 m k_{r \sigma}}{\kappa_{0}^{2}+m^{2} k_{r \sigma}^{2}} \frac{e^{-2 \kappa_{0} w_{r}}}{\kappa_{0} w_{r}},
$$

where $\kappa_{0}=\left[2 m \phi / \hbar^{2}\right]^{1 / 2}$ and $m$ is the effective electron mass in the barrier. Note that $\Gamma_{l \sigma} \neq \Gamma_{r \sigma}$ when the states are not located in the center of the barrier $\left(w_{l} \neq w_{r}\right)$, or when the electrodes are dissimilar $\left(k_{l \sigma} \neq k_{r \sigma}\right)$.

We will calculate the conductance for a barrier with, at arbitrary location, a thin sheet of dopants having a homogeneous energy distribution of resonant levels with spindependent densities $\nu_{\uparrow}$ and $\nu_{\downarrow}$ (per unit volume and unit energy). We take the spin-up and spin-down directions for the dopants to be fixed and identical to those in the left magnetic electrode, whose magnetization direction is also kept fixed, i.e., only the magnetization of the right electrode is assumed to rotate for the purpose of calculating the magnetoresistance. Replacing the sum in Eq. (1) by an integral, we obtain for the conductance $G_{\sigma}^{P}$ per unit area for parallel electrode magnetizations:

$$
G_{\sigma}^{P}=\frac{e^{2} \pi}{\hbar} \nu_{\sigma}\left(\frac{\Gamma_{l \sigma} \Gamma_{r \sigma}}{\Gamma_{l \sigma}+\Gamma_{r \sigma}}\right) .
$$

The expression for $G_{\sigma}^{A P}$ for antiparallel magnetizations is obtained from Eq. (4) by interchanging $\Gamma_{r \uparrow}$ and $\Gamma_{r \downarrow}$ in each spin channel. The spin-integrated conductances are then $G^{P}$ $=G_{\uparrow}^{P}+G_{\downarrow}^{P} \quad$ and $\quad G^{A P}=G_{\uparrow}^{A P}+G_{\downarrow}^{A P}$. The resulting resonant-tunneling magnetoresistance, here defined as $\mathrm{JMR}^{r e s}=\left(G^{P}-G^{A P}\right) / G^{P}$, is explicitly written as

$$
\mathrm{JMR}^{r e s}=1-\frac{\nu_{\uparrow}\left(\frac{\Gamma_{l \uparrow} \Gamma_{r \downarrow}}{\Gamma_{l \uparrow}+\Gamma_{r \downarrow}}\right)+\nu_{\downarrow}\left(\frac{\Gamma_{l \downarrow} \Gamma_{r \uparrow}}{\Gamma_{l \downarrow}+\Gamma_{r \uparrow}}\right)}{\nu_{\uparrow}\left(\frac{\Gamma_{l \uparrow} \Gamma_{r \uparrow}}{\Gamma_{l \uparrow}+\Gamma_{r \uparrow}}\right)+\nu_{\downarrow}\left(\frac{\Gamma_{l \downarrow} \Gamma_{r \downarrow}}{\Gamma_{l \downarrow}+\Gamma_{r \downarrow}}\right)} .
$$

In the following we will calculate the conductance as well as the magnetoresistance due to resonant tunneling and compare that to the conductance and magnetoresistance due to direct tunneling between the electrodes. For the latter we use expression (3) from Bratkovsky, ${ }^{14}$ taking into account the different definition of $\mathrm{JMR}^{\text {res }}$ used there. Fixed parameters are $\phi=3 \mathrm{eV}, m=0.2$ and the spin-integrated density of barrier states $\nu_{\uparrow}+\nu_{\downarrow}=10^{-5} \AA^{-3} \mathrm{eV}^{-1}$.

\section{RESULTS AND DISCUSSION}

To illustrate the effect of a spin-polarized density of barrier states on the tunnel magnetoresistance, we take two identical ferromagnetic Fe electrodes both with $k_{\uparrow}=1.09 \AA^{-1}$ and $k_{\downarrow}=0.42 \AA^{-1}$, separated by a tunnel barrier of width $w$, in which a sheet of dopants is positioned in the center of the barrier. The result is shown in Fig. 1 with the polarization $P_{i}=\left(\nu_{\uparrow}-\nu_{\downarrow}\right) /\left(\nu_{\uparrow}+\nu_{\downarrow}\right)$ of the barrier states varied from 0 to $100 \%$. The bottom panel shows that for $P_{i}=0$, the JMR due to resonant tunneling is only $12.3 \%$, and thus smaller than the $22.0 \%$ obtained for direct tunneling. In contrast, for $P_{i}$ $=100 \%$ the resonant-tunneling JMR is as large as $35.1 \%$, i.e., larger than that for direct tunneling. The enhancement occurs because resonant states are only available for spin-up electrons from the left electrode. The JMR, however, is not equal to 1 as it is limited by the spin polarization of the right
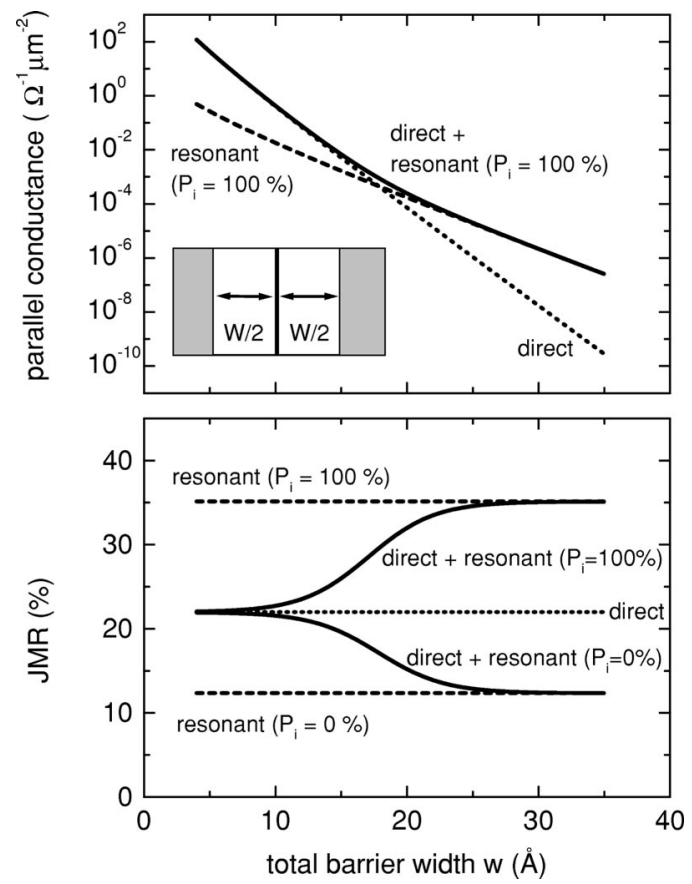

FIG. 1. Parallel conductance (top panel) and magnetoresistance JMR (bottom panel) as function of the width of the tunnel barrier between two identical magnetic $(\mathrm{Fe})$ electrodes, with an ultrathin sheet of resonant levels located in the center of the tunnel barrier. Dotted lines denote direct tunneling between the electrodes, dashed lines the contribution due to resonant tunneling, while solid lines represent the total effect (direct + resonant tunneling). The spin polarization of the resonant states is either 0 or $100 \%$.

electrode. As can be seen in the top panel of Fig. 1, for the chosen density of barrier states, the conductance due to resonant tunneling is smaller than the direct tunneling conductance at small $w$. However, with increasing $w$ the direct tunneling conductance decays faster than resonant tunneling, the latter therefore dominates the total conductance for thick enough barriers. As a result, the total JMR of the junction (direct + resonant tunneling) is enhanced at high barrier thickness for $P_{i}=100 \%$, as shown by the solid line in the bottom panel.

In the previous example we have seen that for $P_{i}$ $=100 \%$, only spin-up electrons from the left electrode are able to contribute to the resonant conductance. The spin polarization of the left electrode is thus irrelevant! As an interesting consequence, resonant tunneling through spinpolarized barrier states exhibits magnetoresistance even when the left electrode is nonmagnetic. This is shown in Fig. 2 where $\mathrm{Au}$ (with $k_{\uparrow}=k_{\downarrow}=1.21 \AA^{-1}$ ) has been used as the left electrode, and the right electrode is $\mathrm{Fe}$ as before. The sheet of dopants has been located at a fixed distance of $15 \AA$ from the right electrode, while $w_{l}$ is varied from 2 to $20 \AA$.

As evidenced by the dashed curve in the bottom panel, the JMR for resonant tunneling is clearly nonzero. In fact, it is exceptionally large at small $w_{l}$. The reason is that for small $w_{l}$, the linewidth $\Gamma_{l \sigma}$ for tunneling from the barrier state to the left electrode becomes much larger than $\Gamma_{r \sigma}$ [see Eqs. (2) and (3)]. Under such conditions, the transitions from the barrier state to the right (magnetic) electrode are rate limiting. Since $P_{i}$ was assumed to be fixed at $100 \%$ and only the magnetization of the right electrode is free to rotate, magne- 


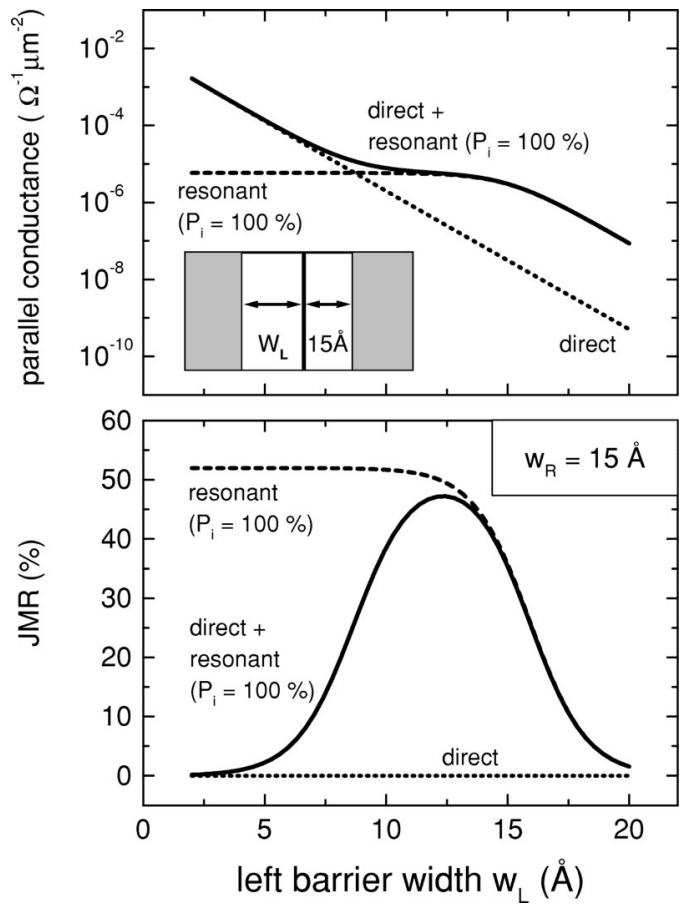

FIG. 2. Parallel conductance (top panel) and JMR (bottom) for a tunnel junction with $\mathrm{Fe}$ as the right electrode and $\mathrm{Au}$ as the left electrode, and a sheet of resonant levels with $100 \%$ spin polarization located at a fixed distance of $15 \AA$ from the right electrode. The left barrier width $w_{l}$ is varied from 2 to $20 \AA$. Dotted lines denote direct tunneling only, dashed lines the contribution due to resonant tunneling, while solid lines represent the total effect (direct + resonant tunneling).

toresistance is produced. For $P_{i}=100 \%$, it is equal to $2 P_{\Gamma_{r}} /\left(1+P_{\Gamma_{r}}\right)$ where $P_{\Gamma_{r}}$ is the spin polarization of $\Gamma_{r \sigma}$ [i.e., $\left.\left(\Gamma_{r \uparrow}-\Gamma_{r \downarrow}\right) /\left(\Gamma_{r \uparrow}+\Gamma_{r \downarrow}\right)\right]$. This explains the large JMR value of $52 \%$ at small $w_{l}$.

At high $w_{l}$, tunneling from barrier states to the left nonmagnetic electrode becomes rate limiting, resulting in a decay of $\mathrm{JMR}^{\text {res }}$. With regard to the conductance, at low $w_{l}$ this is again dominated by direct tunneling, which does not exhibit JMR because the left electrode is nonmagnetic. Hence the net JMR is small. As $w_{l}$ is increased, the total JMR initially goes up because of the increased conductance contribution from the highly polarized resonant tunneling. At high $w_{l}$, resonant tunneling completely dominates, but has a smaller JMR, as explained above. Thus a maximum occurs in the total JMR. For the parameters used here, the optimum total JMR is obtained for $w_{l} \approx 12 \AA$, which is somewhat smaller than $w_{r}=15 \AA$.

The formalism for resonant tunneling can be used to study the effect of a thin sheet of highly spin-polarized dopants in close proximity to a magnetic electrode. To investigate this, suppose we have a tunnel junction with two identical $\mathrm{Fe}$ electrodes and a sheet of $100 \%$ polarized dopants at a distance $w_{l}$ from the left electrode. If the distance is small enough, the dopants may be coupled ferromagnetically or antiferromagnetically to the electrode. The resulting conductance and JMR has been calculated as function of $w_{l}$ for a total barrier width of $20 \AA$ and a total density of barrier states of $10^{-3} \AA^{-3} \mathrm{eV}^{-1}$ (see Fig. 3). As before, the JMR associated with resonant tunneling is much higher than that due
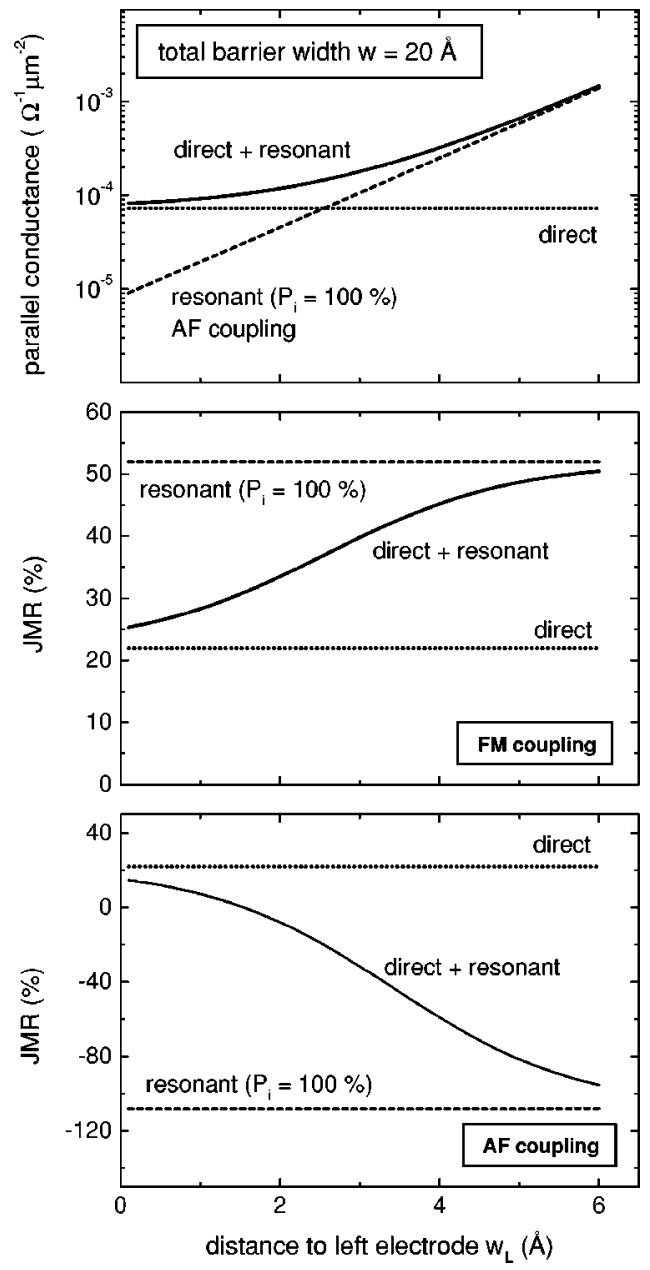

FIG. 3. Parallel conductance (top panel) and JMR (middle and bottom) for a tunnel junction between two identical Fe electrodes, in which a sheet of $100 \%$ spin-polarized dopants is located at a distance $w_{l}$ from the left electrode. For the middle panel, the dopants are coupled ferromagnetically to the left electrode, for the bottom panel the coupling is antiferromagnetic. The total barrier width $w$ is fixed at $20 \AA$.

to direct tunneling. However, in the case of antiferromagnetic coupling the resulting JMR ${ }^{\text {res }}$ is negative, because the sign of $P_{i}$ is opposite to that of the left electrode. In addition, $w_{l}<w_{r}$ such that the dopant-assisted tunneling is determined by the transitions between dopants and the right electrode. The combined effect causes the inversion of the JMR.

Note that in Fig. 3 resonant tunneling has little effect on the total JMR when $w_{l}$ is small, as the resonant conductance is relatively low when states are located far away from the center of the barrier. Improvement is obtained when states are closer to the barrier center (Fig. 3, top panel), although in practice this will also reduce the strength of the magnetic coupling with the electrode, which was not taken into account here.

Experimental observation of the discussed effects in large area magnetic tunnel junctions is not straightforward, because averaging over dopants with randomly oriented spinpolarization will yield no net resonant tunneling magnetoresistance. Hence a ferromagnetically ordered ultrathin film of dopants is required. More practically, at low temperature a magnetic field may be used to align the dopant spins and 
thereby control the polarization $P_{i}$. When one nonmagnetic electrode is used, the existence of barrier states with nonzero spin polarization is unambiguously established if JMR is observed. Structures based on diluted ferromagnetic semiconductors [e.g., (Ga,Mn)As (Ref. 19)] may also be employed, taking advantage of the high level of growth control.

\section{CONCLUSION}

We have analyzed resonant tunneling through spinpolarized barrier levels in a magnetic tunnel junction. For highly polarized barrier states, the tunnel magnetoresistance was shown to be enhanced. Also, it is predicted that whenever the states have a nonzero polarization, magnetoresis- tance should occur if only one electrode is magnetic and the other is not. For a thin sheet of spin-polarized dopants in close proximity to and magnetically coupled to one of the electrodes, we found that the JMR can be enhanced, reduced and, for antiferromagnetic coupling, may be reversed.

\section{ACKNOWLEDGMENTS}

Authors thank J. S. Moodera and A. M. Bratkovsky for the stimulating and fruitful discussions that have triggered the present work. This work was supported by the European Community [Grant No. MEL-ARI ESPRIT 23.307 (SPIDER)].
${ }^{1}$ R. Tsu and L. Esaki, Appl. Phys. Lett. 22, 562 (1973).

${ }^{2}$ L.L. Chang, L. Esaki, and R. Tsu, Appl. Phys. Lett. 24, 593 (1974).

3 S. Datta, Electronic Transport in Mesoscopic Systems (Cambridge University Press, Cambridge, 1995).

4 J.H. Davies, The Physics of Low-dimensional Semiconductors (Cambridge University Press, New York, 1998).

${ }^{5}$ S.J. Bending and M.R. Beasley, Phys. Rev. Lett. 55, 324 (1985).

${ }^{6}$ M. Naito and M.R. Beasley, Phys. Rev. B 35, 2548 (1987).

${ }^{7}$ R. Meservey, P.M. Tedrow, and J.S. Brooks, J. Appl. Phys. 53, 1563 (1982); G.A. Gibson and R. Meservey, ibid. 58, 1584 (1985).

${ }^{8}$ Y. Xu, D. Ephron, and M.R. Beasley, Phys. Rev. B 52, 2843 (1995), and references therein.

${ }^{9}$ L.I. Glazman and K.A. Matveev, Zh. Éksp. Teor. Fiz. 94, 332 (1988) [Sov. Phys. JETP 67, 1276 (1988)].
${ }^{10}$ A.I. Larkin and K.A. Matveev, Zh. Éksp. Teor. Fiz. 93, 1030 (1987) [Sov. Phys. JETP 66, 580 (1987)].

${ }^{11}$ M. Julliere, Phys. Lett. 54A, 225 (1975).

${ }^{12}$ J.S. Moodera, L.R. Kinder, T.M. Wong, and R. Meservey, Phys. Rev. Lett. 74, 3273 (1995); J.S. Moodera and L.R. Kinder, J. Appl. Phys. 79, 4724 (1996).

${ }^{13}$ T. Miyazaki and N. Tezuka, J. Magn. Magn. Mater. 139, L231 (1995).

${ }^{14}$ A.M. Bratkovsky, Phys. Rev. B 56, 2344 (1997).

${ }^{15}$ E. Yu. Tsymbal and D.G. Pettifor, J. Appl. Phys. 85, 5801 (1999).

${ }^{16}$ R. Jansen and J.S. Moodera, J. Appl. Phys. 83, 6682 (1998).

17 R. Jansen and J.S. Moodera (unpublished).

${ }^{18}$ R. Jansen and J.S. Moodera, Appl. Phys. Lett. 75, 400 (1999).

${ }^{19}$ H. Ohno, F. Matsukura, T. Omiya, and N. Akiba, J. Appl. Phys. 85, 4277 (1999). 